\title{
A Preliminary Study on the Management Strategy of University Personnel Files based on Artificial Intelligence Technology
}

\author{
Litao Cui* \\ Zibo Vocational Institute, Zibo 255300, Shandong province, China
}

\begin{abstract}
In order to improve the management strategy for personnel files in colleges and universities, simplify the complex process of file management, and improve file management security and content preservation of the files. This paper elaborates on the application of Artificial Intelligence (AI) technology in university personnel file management through theoretical analysis based on the understanding of AI technology.
\end{abstract}

Keywords: AI technology; Personnel files; Management strategy

Publication date: March, 2021; Publication online: 31 March, 2021

*Corresponding author: Litao Cui, zbvcclt@163.com

\section{Introduction}

Artificial intelligence (AI) was first proposed in 1956. In addition to the development of the times, AI has been applied to various aspects of life and science, also it is called "one of the three cuttingedge technologies in the 21st century." Qin Xia (2020) proposed that the Internet of Things (IoT) technology can be applied to personnel files, and the use of IoT technology can accomplish the intelligent processing of personnel files ${ }^{[1]}$. In the process of managing personnel files, the IoT technology can be used to design a functional system with modules such as label initialization, file receiving, borrowing management, file statistics, user management, system management, and my information, which can improve the management of personnel files. Similarly, Chen Huiming et al (2020) proposed that AI enabled file management can accomplish digitization, meet the needs of social development, and meet the needs of users ${ }^{[2]}$. It can handle a large amount of data and multiple types of file management, and accomplish the simplicity of file management, as well as convenient processing. Fang Binxing et al (2020) proposed that AI empowerment has the function of dissemination, which can enhance the information dissemination power of the society, enhance the ability of the public to obtain information, achieve the efficient matching of information, and provide the possibility for indepth monitoring ${ }^{[3]}$. AI empowerment can meet the needs of file management and achieve the intelligent development of file management. At the same time, Zhang Yang (2020) proposed that Big Data can be used to accomplish the digitization of archives management, cloud storage, data retrieval, data service and information service ${ }^{[4]}$. Furthermore, Yan Guangjun (2019) proposed that the current internet technology archives management still has certain problems and limitations, the security risk factor is high, and the management strategy and management concept needs to be changed to improve the attainment of intelligent file management ${ }^{[5]}$.

\section{Difficulties in Managing the University} Personnel's Files

\subsection{Large amount of information}

At this stage, most colleges and universities have 
various types of file information and data materials, also the content is relatively large as its mainly including digital and image information formats. Although the content of the files includes design and settings, but most universities have organization and management systems to handle it. However, the unregulated standardization of data and information in the archives leads to the confusion, which has a greater impact on the collection and arrangement of archive data by administrators, as well as it increases the difficulty in archives management. This situation has led to a decrease in the efficiency of university management and hindered the sustainable development of university archives management, which is a great obstacle when improving the development of universities.

\subsection{Lack of structure}

The "atypical" phenomenon in the archives of colleges and universities is very severe. Information and data appearing in university personnel files will be summarized in archive records. If archive information cannot be classified and collected accurately, it will be very difficult for employees to search when using archives, thereby reducing the number of archives and the value of data used. The internal information of universities is mainly "unstructured" information, which must be collected and classified for the effective use of archive resource information in enhancing the process of intelligent management.

\subsection{Inability to use invisible information effectively}

Invisible resource information is the main part of personnel files, and the file system is generated as an "obvious information resource." Explicit resources have the characteristics of communication, exchange and expression, and are relatively easy to obtain. Retrieving invisible information from the archives is relatively difficult. Most invisible data is mainly collected based on the independent judgment and work experience of the management. It has the characteristics of independence and exclusivity, and it cannot use objective data for display and expression, as well as information sharing is very difficult.

\section{AI Technology Applications in Personnel File Management}

\subsection{Rapid information collection}

Traditionally personnel files were mainly recorded on paper, which not only took a lot of space, but is also inconvenient to store and carry. In the rapid development of artificial intelligence, the task of file management has shifted from paperless to automated. The achievement of automation effectively shortens the working time and improves the efficiency of file management in a specific way. Artificial intelligence is capable of storing and collecting information, thus it is widely used in document management, reembedding classified paper documents and finally generating electronic documents. In the information era, using intelligent technologies is one of the effective ways to improve the efficiency of file management. For example, intelligent technology for execution and learning, as well as various types of intelligent technologies. Apply intelligent themes to your archive collection with powerful self-control, initiative and adaptability. However, there are huge differences between intelligent themes and other themes. Therefore, administrators need to apply each theme in a unified manner so that they really have the ability to process and collect files. In the era of internet, the application of artificial intelligence in file management will be further developed. The application of AI technology is mainly based on intelligent technology learning. Big Data training can lead to deep learning, and summarizing the laws and knowledge of computer applications. In file management operations, global quantification of file information is the main prerequisite for the application of AI. Administrators need to build network systems through AI, improve network system services, and ensure that network systems can explicitly fulfill users' needs. 


\subsection{Improve security}

Protecting files is a basic task, and only protected files can lay the foundation for future work. In order to ensure the strong security management of file, the administrator can use smart technologies such as voice and fingerprint to complete this operation, so that they can easily retrieve and return data, and provide strong technical support to improve work efficiency. Currently, the AI surveillance system is constantly being optimized and improved. The upgraded system will detect video images and send alert signals to constantly remind administrators, thereby they can increase their awareness of security precautions and propose solutions when security alert occur. No accidents such as information leakage and file loss will occur, personal information can be fully protected, and AI will automatically set the optimal design and settings for file storage to ensure that external factors will not damage the files stored in the computers. Currently, the management department mainly uses firewalls and monitoring systems to complete the protection work and the detection system can monitor illegal intrusions in real-time and play a protective role. The firewall integrates a variety of technologies to analyze and block insecure access in real time, thereby promoting effective database management. In most cases, firewalls will block viruses and ensure that the file system is not damaged by external attacks. In addition, the firewall can attain real-time monitoring and search for damaged or attacked data perfectly.

\subsection{Intelligent file classification}

Intelligent file classification mainly refers to the intelligent processing of classifying and sorting files based on smart technologies such as machine learning and natural language processing to group and collect files to achieve the purpose of efficient and standardized substantial file classification. In the file classification phase, the system is trained and modeled through a large number of classified files, and then the model is used to analyze the content of the archive files and determine the classification number and retention period of the file. In the classification stage, the system automatically classifies the year, retention period, classification number, creation date and other fields of information according to the classification guidelines. In the grouping and packing stage, the system sets the packing guidelines (year, retention period, classification number) and saves the pages at the time of the last change of the files, and waits for the folder grouping operation to be automatically completed.

\subsection{Intelligent file retrieval}

Intelligent file retrieval mainly refers to improving the real-time and accuracy of archive retrieval with the help of OCR recognition, speech recognition, image recognition, machine learning and natural language processing technologies to facilitate the access and acquisition of archive resources. The biggest difference between intelligent retrieval and traditional information retrieval is that the function of intelligent retrieval includes intelligent semantic retrieval, and the retrieval results support the authorizationcontrolled intelligent retrieval process, as shown in Figure 1.

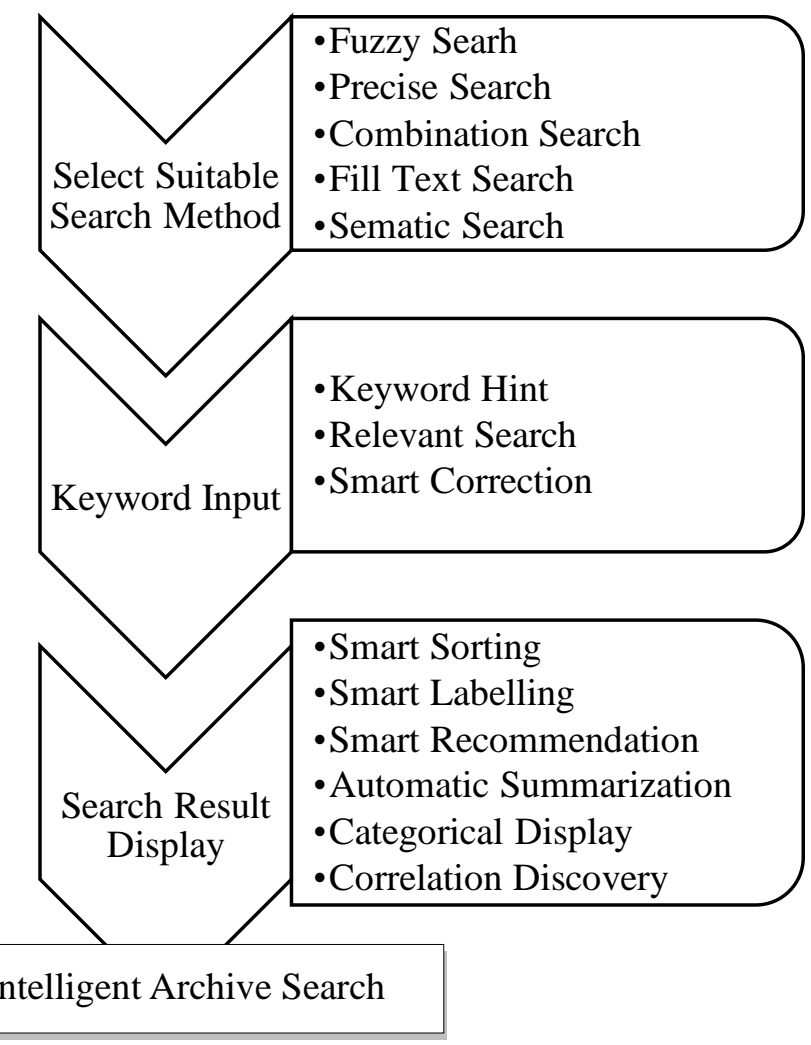

Figure 1. Search process flow 


\section{Conclusions}

In conclusion, due to the in-depth development and application of AI, the field of archivology is inevitably involved in the trend of AI development. The archiving scene will provide a more colorful technical picture and provide new opportunities for solving the problems in the archival work. Although running AI-supported files is favored by most enterprises and universities, but in general the application of AI technology in China's personnel file management is still in the experimental and exploratory stage, and there is still a lack of cases of industry-level application. Based on this phenomenon, archive management in the information age must be solved by technological changes. The archives develop from information, accelerate the application and expansion of related data resources, improve the evolution from "single data source" to "multiple data sources," updates concepts, actively expands relevant technical capabilities, as well as strengthen and track the development of "cognitive intelligent technology." Transition to "cognitive intelligence technology," focuses on practice, actively introducing and exploring related application scenarios, attaining the upgrade from "intelligentization of specific archive links" to "intelligentization of the entire archival process." Additionally, enhance collaboration and establish related improvement collaboration system to open up the scenario from "single-theme exploration" to "multiple-theme collaboration."

\section{Disclosure statement}

The author declares no conflict of interest.

\section{References}

[1]Qin X (2020), Research and Development of the Integrated Management System of Internet of Things Technology Archives Information [J]. Shaanxi Archives, (04):29-31.

[2]Chen H, SHI A, Wang N, Jin W (2020), Discussion on the Application and Development of Artificial Intelligence Technology in Archives Work [J]. China Archives, (03):72-74.

[3]Fang BX, QI JY (2020), AI Technology Empowered Intelligent Communication [N]. China Science Daily, viewed on 2020-07-23(003). [4]Zhang Y (2020). Discussion on Related Issues of File Management Based on Big Data Information Technology [J]. Information Recording Materials, 21(02):47-48.

[5]Yan GJ (2019), My Humble Opinions on File Management Based on Internet Technology [J]. Science and Technology Innovation Herald, 16(31):184-185. 